\title{
A NOVEL CLINICAL SOFTWARE TO ASSESS BONE TISSUE FAILURE RISK USING THE FINITE ELEMENT METHOD COMBINED WITH A MEDICAL RADIOGRAPHY
}

\section{J. Belinha ${ }^{1,2}$, Guilherme F. Henriques ${ }^{2,4}$, A.F.Oliveira ${ }^{3}$ and R.M. Natal Jorge ${ }^{4}$}

\begin{abstract}
${ }^{1}$ Mechanical Engineering Department, School of Engineering, Polytechnic of Porto (ISEP), Porto, Portugal, job@isep.ipp.pt ${ }^{2}$ Institute of Science and Innovation in Mechanical and Industrial Engineering (INEGI), Porto, Portugal, up201306461@fe.up.pt

${ }^{3}$ Head of Orthopedics, Hospital Santo António - Hospital Center of Porto, Porto, Portugal, afonsecaoliveira1@gmail.com

${ }^{4}$ Mechanical Engineering Department, Faculty of Engineering of the University of Porto (FEUP), Porto, Portugal, rnatal@fe.up.pt
\end{abstract}

Introduction: Today, it is commonly accepted by the medical community that radiography is an efficient imaging technique to diagnose bone tumors. Although radiologists are capable to recognize such pathology, to them it is not clear if and how bone tumors influence bone tissue structural response.

Objectives: The main goal of this work is to provide a novel and user friendly clinical software capable to automatically read medical radiographies of femur bones and deliver its failure risk, regardless the pathology (bone tumor, osteoporosis, etc.).

Methods: First, in order to automatically recognize from the radiography the femur contour and domain, a new image segmentation technique was developed. The technique allows to build a computational model of the femur bone and simultaneously attribute mechanical properties to each point of the model (based on the grayscale of the radiography). Then, the virtual model is analyzed with the finite element method and the stress field is obtained, allowing to measure bone failure risk.

Results: The software was tested by trainee engineers and orthopedists using several clinical cases. The software was capable to consistently identify the femur bone domain and provide its failure risk, as shown in figure 1. The results were in agreement with more complex computational mechanics analyses.

Conclusions: Since the developed software is an ongoing project, it allows recurring improvements and enhancements. The feedback provided by the testers allowed to improve significantly the software interface and usability. The robustness and consistency of the obtained results shows that the developed software has the potential to assist the clinical practice.
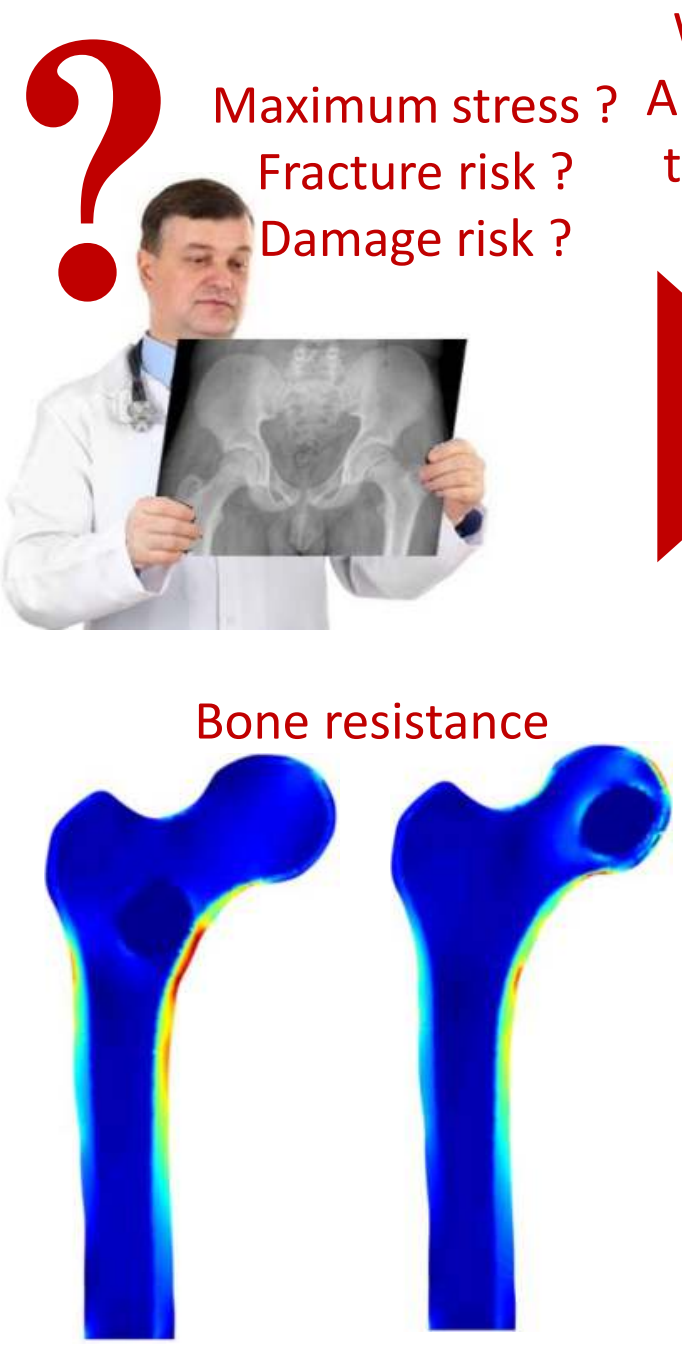

Next APPs

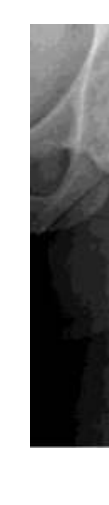

Acknowledges: The authors truly acknowledge the funding provided by Ministério da Ciência, Tecnologia e Ensino Superior - Fundação para a Ciência e a Tecnologia (Portugal), under grants:
SFRH/BPD/111020/2015 and by project funding MIT-EXPL/ISF/0084/2017. Additionally, the authors gratefully acknowledge the funding of Project NORTE-01-0145-FEDER-000022 SciTech - Science and Technology for Competitive and Sustainable Industries, co-financed by Programa Operacional Regional do Norte (NORTE2020), through Fundo Europeu de Desenvolvimento Regional (FEDER).

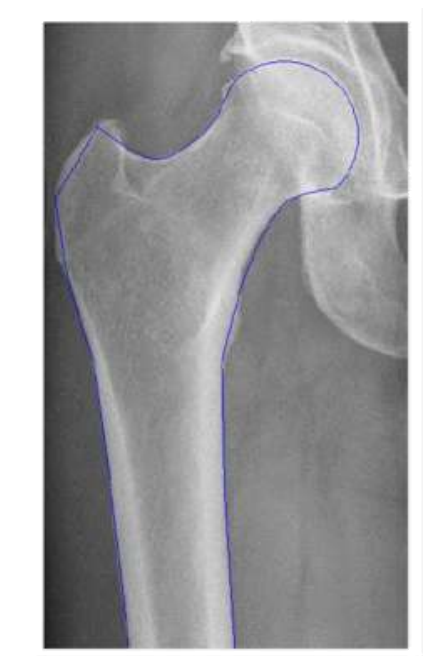

What if it was possible to have a simple

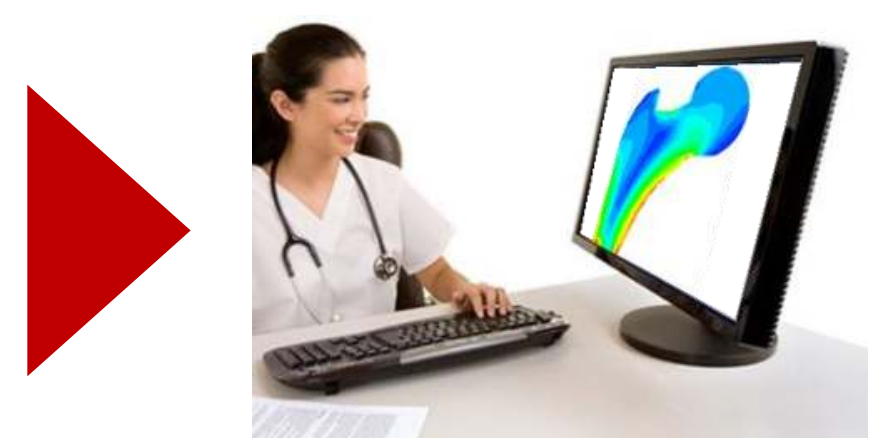

Ongoing
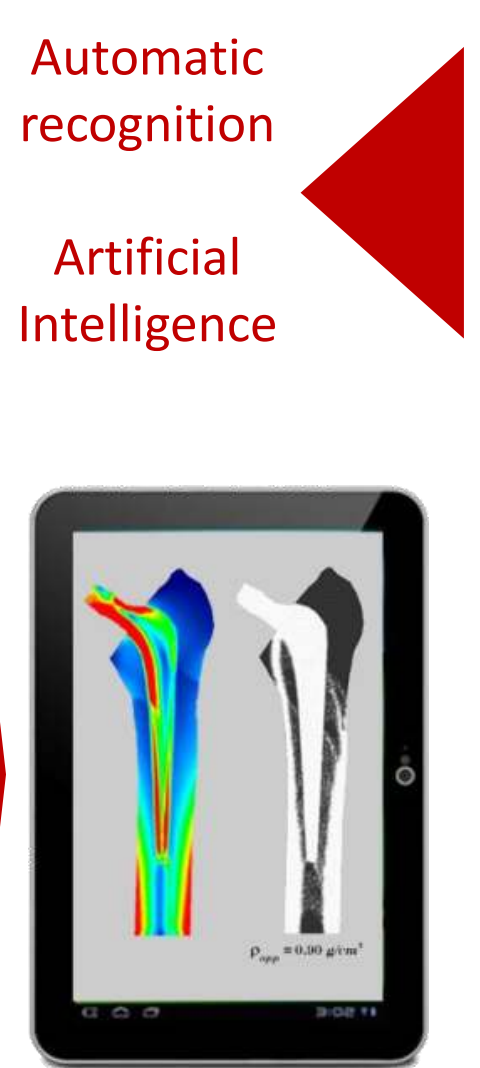

COMPUTATIONAL MECHANICS

cmech.webs.com
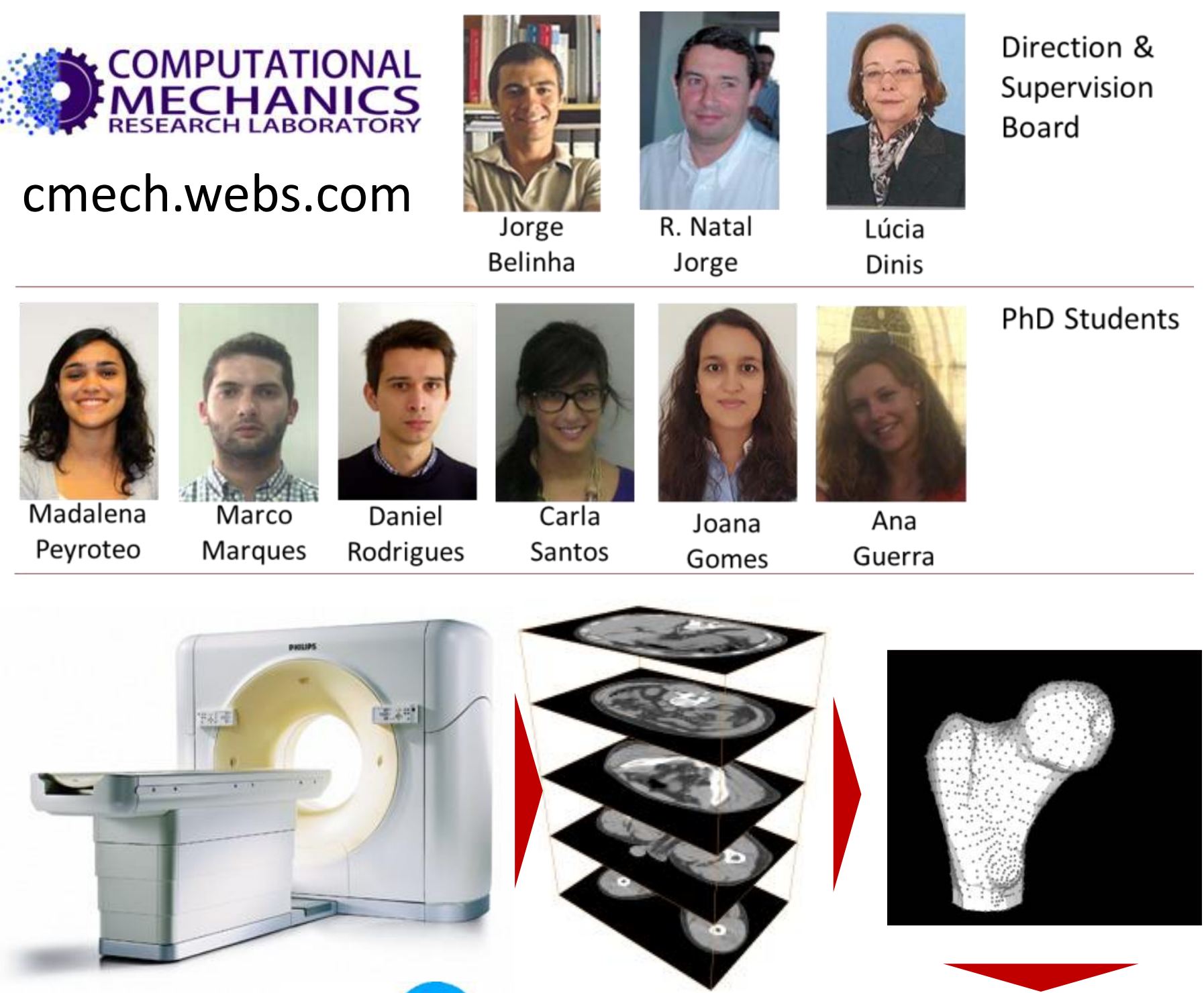

displacement field

stress field

strain field

density field

etc...
Direction \& Supervision Board

PhD Students

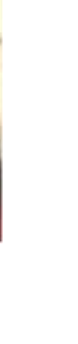

